\title{
Écrire l'histoire
}

Histoire, Littérature, Esthétique

16 | 2016

Accélérations

\section{Les lois mémorielles en Russie et en Ukraine : une histoire croisée}

\section{Nikolay Koposov}

\section{(2) OpenEdition \\ 12 Journals}

Édition électronique

URL : http://journals.openedition.org/elh/1141

DOI : $10.4000 /$ elh. 1141

ISSN : 2492-7457

Éditeur

CNRS Éditions

\section{Édition imprimée}

Date de publication : 15 septembre 2016

Pagination : 251-256

ISBN : 978-2-271-09325-7

ISSN : $1967-7499$

\section{Référence électronique}

Nikolay Koposov, "Les lois mémorielles en Russie et en Ukraine : une histoire croisée », Écrire l'histoire [En ligne], 16 | 2016, mis en ligne le 15 septembre 2019, consulté le 23 septembre 2020. URL : http:// journals.openedition.org/elh/1141; DOI : https://doi.org/10.4000/elh.1141 


\section{Les lois mémorielles en Russie et en Ulkraine : une histoire croisée}

La «révolution de la dignité» en Ukraine en 2014 et l'«étrange guerre» qui s'est déclenchée par la suite entre la Russie et l'Ukraine ont donné lieu à l'adoption dans ces deux pays de lois mémorielles. Cela ne paraît pas surprenant si l'on songe à la longue "guerre de mémoire» entre la Russie de Poutine et les pays voisins, qui culmine avec ces événements. Les lois criminalisant certains énoncés du passé figurent parmi les outils privilégiés de cette guerre.

L'adoption de lois mémorielles en Ukraine et en Russie en 2014 et 2015 a été préparée par plusieurs initiatives législatives, pour la plupart restées sans lendemain, dans les années 1990 et 2000. En dépouillant des archives électroniques de la Rada (le Parlement ukrainien), j'ai trouvé plus de quatre-vingt-dix projets renvoyant à des questions de mémoire historique, dont une dizaine seulement sont devenus des lois. En Russie, où l'espace politique est, depuis 2000, strictement contrôlé par le Kremlin, le nombre des initiatives similaires n'a pas dépassé une trentaine, ce qui est cependant beaucoup par comparaison avec les pays occidentaux.

Dans les années 1990, les élites ukrainiennes n'utilisaient le passé dans des buts politiques qu'avec précaution, pour éviter des conflits à l'intérieur d'un pays dont la mémoire historique était éminemment plurielle. Le clivage principal séparait l'Est industriel et russophone de l'Ouest traditionnellement opposé à la russification poursuivie par l'Empire russe et l'Union soviétique. Les mesures législatives adoptées en Ukraine à l'égard de la mémoire historique se limitaient alors, pour l'essentiel, à la réhabilitation des victimes des répressions staliniennes. En Russie, une loi similaire a été adoptée au début des années 1990. Un peu plus tard, les débats acharnés sur le passé qui divisaient le gouvernement démocratique et l'opposition communiste et nationaliste ont donné lieu à toute une série de projets tendant à réprimer des activités considérées comme néofascistes, incluant la négation des crimes contre l'humanité. Douze projets «antifascistes» ayant un aspect de loi 
mémorielle ont été proposés au Parlement russe entre 1995 et 1999.

À partir de 1995, la notion de loi mémorielle devint ainsi populaire parmi les politiciens démocrates russes, dans le contexte de la consolidation et des succès électoraux du mouvement nationaliste. De leur point de vue, pour arrêter l'essor de l'«extrémisme politique», il fallait criminaliser la négation des crimes des régimes totalitaires, y compris la glorification de Staline, dont l'image servait de symbole à l'opposition communiste et nationaliste. Ce modèle que les démocrates russes auraient souhaité imposer au Parlement a été par la suite adopté en Pologne, où la loi de 1998 punit la négation des crimes communistes et fascistes. Ce texte a servi de modèle à de nombreuses lois adoptées dans les pays autrefois dits «de l'Est» ou d'anciennes républiques soviétiques, en particulier l'Ukraine et la Lituanie. Quant aux communistes et aux nationalistes russes des années 1990, ils ne pouvaient ni abandonner le territoire de l'antifascisme aux démocrates ni criminaliser la glorification du fascisme (pour ne pas déplaire à leurs alliés d'extrême droite). Ils proposaient donc d'introduire des sanctions administratives pour la "propagande du fascisme». Mais aucun de ces projets n'a été adopté.

Dans les années 2000, la politique de l'histoire menée par le gouvernement russe a radicalement changé après la formation du régime autoritaire et nationaliste de Vladimir Poutine. Le culte de la mémoire de la Grande Guerre patriotique (le nom sous lequel la Seconde Guerre mondiale est connue en Russie) est devenu le mythe fondateur de la Russie poutinienne. Ce mythe sert à consolider le soutien politique au régime à l'intérieur du pays et participe au contrôle qui continue d'être revendiqué sur l'Europe de l'Est, considérée comme «zone d'influence» de la Russie. La rhétorique néo-impérialiste du gouvernement russe a provoqué des conflits mémoriels à l'intérieur des pays de l'Est aussi bien qu'entre ceux-ci et la Russie. Les années 2000 ont ainsi vu se déclencher plusieurs «guerres de mémoire» en Europe de l'Est.

La propagande russe a rencontré un fort écho parmi les minorités russophones, surtout en Ukraine, que le Kremlin considérait comme une clé de l'Europe de l'Est. L'objectif de Moscou consistait à utiliser les conflits mémoriels pour diviser la société ukrainienne. Surtout depuis la «révolution orange» de 2004, qui a été provoquée par une tentative du Kremlin pour établir son contrôle sur Kiev, les politiciens et les médias russes n'ont pas ménagé leurs efforts pour populariser, en Ukraine et ailleurs, l'image de l'Ukraine comme un failed state, comme un pays intérieurement divisé au point de n'être plus gouvernable (Vladimir Poutine lui-même aurait déclaré à George Bush en 2008: «L'Ukraine n'est même pas un État»). Les nationalistes russes et les politiciens pro-russes en Ukraine sont devenus le moteur des guerres de mémoire en Ukraine et entre l'Ukraine et la Russie.

Avant même la révolution orange, ces groupes s'étaient appliqués à promouvoir en Ukraine le mythe russe de la Seconde Guerre mondiale. Dans le cadre de ce mythe, les partisans de l'indépendance ukrainienne apparaissaient comme les "alliés du nazisme», selon la vieille formule stalinienne: "Celui qui est contre le communisme [1'URSS puis la Russie en l'occurrence] est pour 
le fascisme.» Le fait avéré que, pendant la Seconde Guerre mondiale, une partie des nationalistes ukrainiens s'étaient alliés, au moins provisoirement, avec l'Allemagne nazie, a été utilisé pour compromettre le mouvement ukrainien de libération nationale en général.

Le président Viktor Iouchtchenko, arrivé au pouvoir à la suite de la révolution orange, a pris l'initiative d'une «contre-offensive mémorielle». Sa politique consistait à promouvoir la mémoire de $l^{\prime}$ Holodomor («extermination par la famine", c'est-à-dire la famine artificielle organisée par le régime stalinien en Ukraine en 1932-1933) au rang de mythe unificateur national. Il a proposé toute une série d'initiatives législatives ayant pour but de criminaliser la négation de $l^{\prime}$ Holodomor "comme un génocide du peuple ukrainien». Une référence aux lois mémorielles européennes servait à justifier cette démarche. Mais si la Rada a alors reconnu l'Holodomor comme un génocide, elle n'a pas criminalisé sa négation (loi $\mathrm{n}^{\circ} 376-\mathrm{V}$ du 28 novembre 2006).

La bataille autour de la mémoire de l'Holodomor a pris une dimension internationale: plusieurs pays ont reconnu $l^{\prime}$ Holodomor comme un génocide, tandis que certains autres, tout particulièrement la Russie, ont refusé de le faire. Le président russe Dmitri Medvedev a même adressé en novembre 2008 une lettre à son collègue ukrainien disant que l'Holodomor avait été un crime du régime stalinien contre les peuples de l'URSS, et non pas un crime de la Russie contre l'Ukraine.

Les initiatives mémorielles de Iouchtchenko ont déclenché une guerre des lois dans laquelle les partisans du «choix européen» et les nationalistes ukrainiens se sont confrontés avec les communistes, les radicaux pro-Kremlin et les politiciens modérés (dont certains activistes juifs), effrayés par la montée du nationalisme ukrainien. En effet, les tentatives pour promouvoir, surtout dans l'Ukraine de l'Ouest, le culte de Stepan Bandera, qui fut pendant la Seconde Guerre mondiale le leader de l'Organisation des nationalistes ukrainiens (OUN), et d'autres leaders nationalistes des années 1940, ne pouvaient que ressusciter les mémoires de l'extermination des Juifs, à laquelle certains nationalistes ukrainiens avaient activement participé.

Chaque "parti » a présenté une quarantaine de projets, dont certains formaient des "chaînes ": chaque fois qu'un parti déposait un projet de loi - tendant par exemple à pénaliser la profanation des tombes des soldats soviétiques - apparaissait un projet symétrique, tendant par exemple à criminaliser la profanation des tombes des "combattants pour l'indépendance de l'Ukraine» (membres de l'Armée insurrectionnelle ukrainienne - UPA, dont Bandera avait également été l'un des dirigeants). En gros, les projets «pro-russes» visaient à pénaliser la «réhabilitation du nazisme» et la profanation des monuments commémoratifs de la Seconde Guerre mondiale, tandis que les projets pro-européens ou nationalistes visaient à bannir les «idéologies totalitaires", à criminaliser la négation de l'Holodomor (et, parfois, de l'Holocauste) et à réhabiliter l'UPA et d'autres organisations nationalistes des années 1940.

De nouveaux projets russes sont apparus dans le contexte des conflits mémoriels avec l'Ukraine aussi bien qu'avec la Pologne et les pays baltes, tels que la confrontation entre Moscou et Tallinn 
après le déplacement du monument aux soldats soviétiques du centre de la capitale estonienne vers un cimetière militaire en 2007. Leur objectif était de criminaliser la «négation de la victoire de l'URSS dans la Seconde Guerre mondiale», la «réhabilitation du nazisme» et «l'héroïsation des collaborateurs du nazisme». La terminologie utilisée dans ces projets - aussi bien que dans certains projets ukrainiens - était élaborée dans le milieu russo-ukrainien des activistes de la politique de la mémoire. Ainsi, le premier projet où figurait la formule «la réhabilitation du nazisme et l'héroïsation des collaborateurs du nazisme» a été présenté à la Rada par Vadim Kolesnitchenko en janvier 2009. Kolesnitchenko était un partisan radical du «choix russe» et membre du Parti des régions de Viktor Ianoukovitch. C'est lui qui, en janvier 2014, a été à l'origine de l'adoption des «lois dictatoriales». Mais la même formule a été employée dans le projet de Konstantin Zatouline, un nationaliste russe à qui ses activités en Ukraine ont coûté depuis 2004 plusieurs interdictions d'entrée sur ce territoire. Zatouline a commencé à travailler sur son projet en décembre 2008, et celui-ci a été publié en avril 2009.

Mais les autorités russes n'ont pas soutenu l'initiative de Zatouline. Elles ont préféré présenter leur propre projet (la «loi Iarovaïa»), qui criminalisait la «négation publique des crimes nazis» aussi bien que les «accusations visant à qualifier de criminelle toute action entreprise par un État membre de la coalition anti-hitlérienne » (y compris donc l'URSS de Staline).

La «détente» dans les relations de la Russie avec l'Occident qui a suivi la visite de Barack Obama à Moscou en juillet 2009 a créé une situation politique défavorable à l'adoption de cette loi. Les autorités russes ne sont revenues à ce projet qu'en 2013, après le tournant conservateur radical dans la politique de Poutine. En 2014, la nouvelle «guerre froide» entre la Russie et l'Occident autour de la crise en Ukraine a abouti à l'adoption des lois criminalisant certaines énonciations sur le passé dans les deux pays.

Deux lois mémorielles ont fait partie $\mathrm{du}$ «paquet» des lois dictatoriales du 16 janvier 2014 imposées à la Rada par les partisans de Ianoukovitch. Toutes deux ont été présentées par le leader des communistes ukrainiens Petro Simonenko. Elles n'étaient pas nouvelles: la première loi (loi no 729-VII du 16 janvier 2014) criminalisant la négation ou la justification des crimes fascistes était la cinquième version du projet de juillet 2009, qui avait reproduit le projet russe de mai 2009 (la première version de la loi Iarovaïa). La deuxième loi ukrainienne de janvier 2014 criminalisait la profanation des monuments de guerre (loi n ${ }^{\circ} 728$-VII du 16 janvier 2014); elle était également la cinquième version d'un projet datant de 2009.

Ces lois dictatoriales ont été abrogées par la Rada le 28 janvier 2014. Mais, le même jour, le Parlement ukrainien a de nouveau voté en faveur des lois Simonenko, qui sont restées en vigueur jusqu'en avril 2015. La raison en était simple: l'opposition démocratique et nationaliste, dont la priorité était de créer une coalition anti-Ianoukovitch aussi large que possible, a choisi de ne pas provoquer de nouveaux conflits mémoriels.

La Douma (la chambre basse du Parlement russe) a adopté la troisième version de la loi Iarovaïa en avril 2014, au 
lendemain de l'occupation de la Crimée. Pourtant, son adoption a été précédée par la compétition, inédite en Russie, entre deux projets alternatifs dont chacun a été soutenu par l'une des chambres du Parlement russe. Le projet Iarovaïa avait le soutien de la Douma, tandis que le Conseil de la Fédération était en faveur du projet de Boris Spiegel, le président adjoint de son Comité pour la législation. Spiegel, un oligarque russe d'origine ukrainienne, avait été le président du Congrès international des communautés juives russophones et le fondateur du mouvement international «Le monde sans nazisme». Sa stratégie consistait à mobiliser l'opinion internationale contre les nationalistes en Ukraine et dans les pays baltes. Dans ce but, il proposait de criminaliser non seulement la réhabilitation du nazisme en général, mais aussi la négation de l'Holocauste. En d'autres mots, il voulait réunir le mythe russe de la guerre avec la mémoire européenne de l'Holocauste. Mais cela risquait de compromettre la "pureté» du mythe russe, selon lequel le peuple russe avait été la victime principale d'Hitler. Remontant à l'époque de la guerre et du procès de Nuremberg, quand l'Holocauste n'avait pas encore été reconnu par la communauté internationale comme le principal crime nazi, ce mythe considérait le nazisme comme une manifestation $\mathrm{du}$ militarisme et de $l^{\prime}$ «impérialisme» allemands - et, plus généralement, occidental - dirigés contre la Russie. C'est pour cela que, dans les conditions de la confrontation avec l'Occident, les autorités russes ont préféré soutenir la loi Iarovaïa, qui défendait le mythe russe dans ses formes classiques.

La version définitive de la loi russe (loi $\mathrm{n}^{\circ}$ 332-FZ du 4 avril 2014) criminalise «la négation des faits, établis par le verdict du Tribunal [de Nuremberg], l'approbation des crimes établis par ledit verdict, ainsi que la diffusion de fausses informations concernant les actions de l'URSS pendant la Seconde Guerre mondiale». À la différence des lois européennes, qui protègent les mémoires des victimes des génocides, la loi russe défend la mémoire du régime stalinien. À ma connaissance, personne n'a encore été condamné en application de cette loi, mais elle est souvent évoquée dans les débats publics pour menacer ceux qui mettent en doute l'idéologie quasi officielle de la Russie de Poutine.

La crise ukrainienne s'est déroulée d'une façon que le Kremlin n'avait pas prévue: l'agression russe a abouti au rassemblement de la société ukrainienne. Dans ces conditions, le nouveau régime a osé, en avril 2015, abroger les lois Simonenko et adopter des lois mémorielles alternatives (connues comme les «lois de décommunisation»). Ces quatre lois ( ${ }^{\text {os }}$ 316-VIII, 314-VIII, 315-VIII et 317-VIII du 9 avril 2015) sont également issues des combats législatifs des années précédentes: la plupart de leurs propositions avaient été formulées en 2005 par Oleg Tiagnibok, le leader du parti ultranationaliste Svoboda («Liberté»).

Les lois de «décommunisation» prévoient la destruction des monuments de l'époque communiste; l'ouverture des archives des services secrets soviétiques; la prohibition de la négation du caractère criminel des régimes fascistes et communistes (aucune sanction spéciale n'a cependant été prévue); la criminalisation de l'utilisation des symboles fascistes et communistes (passible de cinq ans de détention, et de dix ans en cas de «circonstances aggravantes»); 
et la réhabilitation sans condition des "combattants pour la libération de l'Ukraine» (qui jusqu'en 2015 ne pouvaient être réhabilités que s'ils n'avaient pas commis de crimes contre l'humanité). L'une des lois mémorielles visait toutefois à assurer la «perpétuation de la victoire sur le nazisme dans la Seconde Guerre mondiale», ce qui était un geste de conciliation envers les partisans du mythe russe. Mais cette loi se fonde sur une conception de la guerre bien différente de celle qui est généralement admise en Russie: elle met l'accent non pas sur la victoire de l'URSS, mais sur la victoire sur le nazisme, aussi bien que sur les souffrances de toutes les victimes de la guerre, des soldats soviétiques aux membres de l'OUN et aux victimes du génocide nazi. La «lustration symbolique» (pour citer le projet de loi de Tiagnibok de 2005), y compris la destruction massive des statues de Lénine («la peste des Lénine», ou léninopad), a été la première conséquence pratique de l'adoption des lois de décommunisation.
Il est évident que les lois mémorielles, dont l'objectif initial en Occident était de promouvoir une culture démocratique de la mémoire mettant l'accent sur les souffrances des victimes, ont fini par devenir, surtout dans l'est du continent, des moyens privilégiés de la mobilisation nationaliste et des guerres de mémoire. Cette évolution est bien sûr due aux particularités des pays ayant connu le régime socialiste soviétique, et le principe abusus non tollit usum semble, dans une certaine mesure, applicable ici. Mais ces évolutions sont aussi liées au principe même de l'utilisation de la loi criminelle comme instrument de la régulation de la mémoire historique. La vérité de la loi est toujours très réductrice en comparaison avec la vérité historique, bien plus complexe et nuancée. Les lois mémorielles donnent aux nationalistes et aux populistes un instrument trop commode pour manipuler les faits du passé, ce qui diminue nos chances de parvenir à une compréhension de l'histoire fondée sur son examen critique. 